\title{
ON THE PUNCTUAL AND LOCAL ERGODIC THEOREM FOR NONPOSITIVE POWER BOUNDED OPERATORS
}

IN $L_{\mathbf{C}}^{p}[0,1], 1<p<+\infty$

\section{ASSANI}

\begin{abstract}
We show in this note that there exists a function $f \in \bigcap_{1<p<+\infty} L_{\mathbf{C}}^{p}[0,1]$ and for each $p$ an isomorphism $T: L_{\mathbf{C}}^{p} \rightarrow L_{\mathbf{C}}^{p}$ such that $\sup _{n \in \mathbf{Z}}\left\|T^{n}\right\|<+\infty$ and $T$ does not satisfy the punctual ergodic theorem.

We give also an example of a one-parameter semigroup $\left(T_{t}, t \geqslant 0\right)$ of power bounded operators in each $L_{\mathbf{C}}^{p}(1<p<+\infty)$ for which the assertion of the local ergodic theorem $\left((1 / t) \int_{0}^{t} T_{s} f d s\right.$ converge almost everywhere as $t \rightarrow 0_{+}$for all $f \in L^{p}$ ) fails to be true.
\end{abstract}

1. Introduction, definitions and notations. Let $(\Omega, \mathscr{A}, P)$ be a $\sigma$ finite measure space and $L^{p}(\Omega, \mathscr{A}, P)(1<p<+\infty)$ the Banach space of complex valued measurable functions on $\Omega$ such that $\|f\|^{p}=\int|f(x)|^{p} d \mu<+\infty$. An operator $T$ : $L^{p}(\Omega, \mathscr{A}, P) \rightarrow L^{p}(\Omega, \mathscr{A}, P)$ satisfies the punctual ergodic theorem (p.e.t.) if for each $f \in L^{p}(\Omega, \mathscr{A}, P)$

$$
M_{n}(T) f=\frac{1}{n} \sum_{i=0}^{n-1} T^{i} f
$$

is a.e. convergent in $\Omega$.

It is known that $T$ satisfies the punctual ergodic theorem in the following cases: for $1<p<\infty, p \neq 2$ and $T$ an isometry (for an invertible isometry, A. Ionescu Tulcea [1] and for a general isometry R. V. Chacon and S. A. McGrath [2]), for $1<p<+\infty$ and $T$ a positive contraction (M. A. Akcoglu [3]) and for $1<p<\infty$ and $T$ an isomorphism such $T$ and $T^{-1}$ are both power bounded and positive (A. de la Torre [4]). D. L. Burkholder [5] constructed a contraction $T: L^{2} \rightarrow L^{2}$ which does not satisfy the punctual ergodic theorem. Akcoglu and Sucheston [6] remarked that $T$ can be geometrically dilated to an isometry on an $L^{2}$ space which does not satisfy the punctual ergodic theorem.

M. Feder [7] constructed for each $1<p \leqslant 2$ an isomorphism $T: L^{p}[0,2] \rightarrow$ $L^{p}[0,2]$, so that $T$ and $T^{-1}$ both are power bounded, and $T$ does not satisfy the p.e.t. In [14] we have shown that for each $1<p<+\infty$ there exists a power bounded operator $T: L_{\mathbf{R}}^{p}[0,1] \rightarrow L_{\mathbf{R}}^{p}[0,1]$ such that $T$ and $T^{*}$ do not satisfy the p.e.t.

In this paper we show first that there exists for each $p, 1<p<+\infty$, an isomorphism $T$ such that $T$ and $T^{-1}$ are both power bounded and $T$ does not satisfy

Received by the editors November 11, 1984.

1980 Mathematics Subject Classification. Primary 47A35; Secondary 46E30.

1986 American Mathematical Society $0002-9939 / 86 \$ 1.00+\$ .25$ per page 
the p.e.t. This result is then extended to some sequences of operators defined by regular sequences $\left(\alpha_{n K}\right)$, introduced in [13].

Definition 1. Let $\left(\alpha_{n K}\right)$ be a sequence of positive numbers. $\left(\alpha_{n K}\right)$ is said to be regular if

(i) $\forall n \in \mathbf{N}, \sum_{K=0}^{\infty} \alpha_{n K}=1$,

(ii) $\forall z \in \mathbf{C},|z| \leqslant 1, z \neq 1,\left|\sum_{K=0}^{\infty} \alpha_{n K} z^{K}\right| \rightarrow 0$,

(iii) $\forall n \in \mathbf{N}$, the radius of convergence $r_{n}{ }^{n}$ of the series $\sum_{K=0}^{\infty} \alpha_{n K} z^{K}$ satisfies the condition $r_{n}>1$.

Let $T_{t}(t \geqslant 0)$ be a strongly continuous one-parameter semigroup of bounded linear operators on $L_{C}^{p}$. This means that

(i) $T_{t}$ is a bounded linear operator on $L^{p}$ for any $t \geqslant 0$,

(ii) $T_{t+s} f=T_{t} \circ T_{s} f$ for any $t, s \geqslant 0$ and $f \in L_{\mathbf{C}}^{p}$,

(iii) $\lim _{t \rightarrow 0_{+}}\left\|T_{t}-T_{s} f\right\|=0$ for any $f \in L_{\mathbf{C}}^{p}$.

We note $S_{t} f=\int_{0}^{t} T_{s} f d s$. Local ergodic theorems assert that $\lim _{t \rightarrow 0_{+}}\left(S_{t} / t\right) f$ exists a.e. for all $f \in L^{p}$. Local ergodic theorems have been studied by many authors (see [8]). We shall adapt the construction given by Akcoglu and Krengel in [9] to get an example of local ergodic divergence for power bounded operators in $L^{p}$ for each $p, 1<p<+\infty$.

II. On the punctual ergodic theorem in $L_{\mathrm{C}}^{p}[0,1], 1<p<+\infty$. Let $\left(\phi_{n}^{K}\right)$ be Haar's system. The functions $\phi_{n}$ are defined by the equations

$$
\phi_{1}(t)=\phi_{0}^{(0)}(t)=1 \text { for } t \in[0,1]
$$

and, for $m=2^{n}+k$ with $1 \leqslant k \leqslant 2^{n}, n=0,1, \ldots$,

$$
\phi_{m}(t)=\phi_{n}^{(K)}(t)= \begin{cases}+\sqrt{2^{n}} & \text { for } t \in\left[\frac{2 K-2}{2^{n-1}}, \frac{2 K-1}{2^{n+1}}\right), \\ -\sqrt{2^{n}} & \text { for } t \in\left(\frac{2 K-1}{2^{n+1}}, \frac{2 K}{2^{n+1}}\right], \\ 0 & \text { elsewhere. }\end{cases}
$$

P. L. Ulyanov [10] has obtained the following result.

THEOREM 1. There exists a function $f \in \bigcap_{1<p<+\infty} L^{p}[0,1], f=\sum_{i=1}^{\infty} a_{i} \phi_{i}$, and a permutation of the integers $\pi$ such that the series $\sum a_{\pi(i)} \phi_{\pi(i)}(t)$ diverges unboundedly on $[0,1]$ almost everywhere.

The first proof given in [10] uses a quite difficult modification of Zahorski's construction. In a recent publication [11] P. L. Ulyanov gives a simpler proof (in $L^{2}$ ). From this article and the arguments of the first paper we can get a simpler proof of this theorem. Olevskii [15] gave also (in $L^{2}$ ) another proof of this result. From this theorem we can get the theorem announced.

THEOREM 2. There exists a function $f \in \bigcap_{1<p<+\infty} L^{p}[0,1]$ and for each $p, 1<p<$ $+\infty$, an isomorphism $T$ such that $T$ and $T^{-1}$ are both power bounded and

$$
M_{n}(T) f=\frac{I+T+T^{2}+\cdots+T^{n-1}}{n} f
$$

does not converge almost surely. 
Proof. Let $p$ be fixed, $1<p<+\infty$. From Haar's system $\left(\phi_{n}^{(K)}\right)=\left(\phi_{m}\right)$ we can get an unconditional base of $L_{C}^{p}[0,1],\left(\tilde{\phi}_{n}^{K}\right)=\left(\tilde{\phi}_{m}\right)$. Consider the permutation $\pi$ and $f$ of the previous theorem; $\left(\tilde{\phi}_{\pi(m)}\right)$ is again an unconditional base of $L_{C}^{p}[0,1]$ and if $f=\sum a_{i} \tilde{\phi}_{i}$ then $f=\sum a_{\pi(i)} \tilde{\phi}_{\pi(i)}$.

By using Remark 1 in [7] we can get an isomorphism $T$ ( $T$ and $T^{-1}$ are both power bounded) and a sequence $n_{1}<n_{2}<\cdots<n_{K}<\cdots$ such that $\| M_{n_{K}}(T)$ $Q_{K} \|<+\infty$, where $Q_{K}$ is the projection

$$
Q_{K}\left(\sum_{i=1}^{\infty} b_{i} \tilde{\phi}_{\pi(i)}\right)=\sum_{i \geqslant K} b_{i} \tilde{\phi}_{\pi(i)} .
$$

We can then deduce easily that the sequence $M_{n_{K}}(T) f$ does not converge almost surely on $[0,1]$. This result extends easily to the case of operators defined by a regular sequence $\left(\alpha_{n K}\right)$ (see Definition 1).

THEOREM 3. Let $\left(\alpha_{n K}\right)$ be a regular sequence and consider the sequence of operators

$$
R_{n}(T)=\sum_{K=0}^{\infty} \alpha_{n K} T^{K}
$$

Then for each $p, 1<p<+\infty$, there exists an isomorphism $T: L^{p} \rightarrow L^{p}$ and $f \in L^{p}$ such that the sequence $R_{n}(T) f$ does not converge almost surely.

Proof. Consider the unconditional base $\left(\tilde{\phi}_{\pi(m)}\right)$ of the previous proof and let $P_{m}$ be the projection on $\phi_{\pi(m)}$. As in [12] the operator $T$ will be diagonal, i.e., $T=\sum_{i=1}^{\infty} \lambda_{i} P_{i}$, where the $\lambda_{i}$ are complex numbers with $\left|\lambda_{i}\right|=1$ and $\lambda_{i} \neq 1, \forall i$. Take $\lambda_{1},\left|\lambda_{1}\right|=1$ and $\lambda_{1} \neq 1$. Choose $\left(\varepsilon_{i}\right)$ such that $\varepsilon_{i}>0$ and $\sum \varepsilon_{i}=1$. There exist an integer $n_{1}$ and a real number $\delta_{1}$ such that $\left|R_{n_{1}}\left(\lambda_{1}\right)\right|<\varepsilon_{1}$ and $\left|R_{n_{1}}(z)-1\right|<\varepsilon_{1}$ if $|z-1|<\delta_{1}$, by the regular properties of the sequence $\left(\alpha_{n K}\right)$.

If $\lambda_{i}, n_{i}, \delta_{i}$ have been chosen for $1 \leqslant i \leqslant j$, then we choose $\lambda_{j+1}$ such that $\left|\lambda_{j+1}-1\right|<\delta_{i}$ for $1 \leqslant i \leqslant j$. Then we take $n_{j+1}>n_{j}$ such that $\left|R_{n_{j+1}}\left(\lambda_{i}\right)\right|<\varepsilon_{j+1}$ for $1 \leqslant i \leqslant j+1$ and $\delta_{j+1}>0$ such that $\left|R_{n_{j+1}}(\lambda)-1\right|<\varepsilon_{j+1}$ when $|\lambda-1|<\delta_{j+1}$.

The end of the proof is then the same as for the previous theorem.

REMARK 4. (1) Theorem 2 shows that the Abel means of an isomorphism $T$ such that $T$ and $T^{-1}$ are power bounded in $L^{p}$ are not always convergent a.e. (take $\left.\alpha_{n K}=(1 / n)(1-1 / n)^{K}\right)$.

(2) An analog proof had been given in the case of contractions of $L^{2}$ in [13].

III. On the local ergodic theorem in $L_{C}^{p}[0,1], 1<p<+\infty$. Let $\left(\tilde{\phi}_{\pi(m)}\right)$ be the unconditional base of the previous section, $V_{k}=I-Q_{K}$, and $p$ fixed, $1<p<+\infty$. We have the following lemma, containing ideas from [9].

LEMMA 5. There exist sequences $\left(\lambda_{n}\right), n=1, \ldots,\left(t_{K}\right), K=1, \ldots$, with $\lambda_{n}>0$ and $0<t_{K} \rightarrow 0$ such that $\Sigma\left\|V_{K}-\left(1 / t_{K}\right) S_{t_{K}}\right\|<+\infty$.

Proof. Choose $\left(\varepsilon_{K}\right) \varepsilon_{K}>0$ such that $\sum \varepsilon_{K}<+\infty$. Then take $\lambda_{1}>0$ arbitrarily and choose $0<t_{1}<1$ so small that

$$
\left|\frac{e^{i \lambda_{1} t_{1}}-1}{i \lambda_{1} t_{1}}-1\right|<\varepsilon_{1} .
$$


If $\lambda_{1}, \lambda_{2}, \ldots, \lambda_{K}$ and $t_{1}, \ldots, t_{K}$ are already chosen, choose $\lambda_{K+1}>0$ sufficiently large so that

$$
\left|\frac{e^{i \lambda_{K+1} t_{m}}-1}{i \lambda_{K+1} t_{m}}\right|<\varepsilon_{m} \quad \text { for each } m=1,2, \ldots, K
$$

Then choose $s<t_{K+1}<1 /(K+1)$ so small that

$$
\left|\frac{e^{i \lambda_{n} t_{K+1}}-1}{i \lambda_{n} t_{K+1}}-1\right|<\varepsilon_{K+1} .
$$

THEOREM 6. Given $p, 1<p<+\infty$, there exists a function $f \in \bigcap_{1<p<+\infty} L{ }^{p}[0,1]$ and a one-parameter semigroup $\left(T_{t}, t \geqslant 0\right)$ of power bounded operators in $L_{\mathbf{C}}^{p}[0,1]$ for which $(1 / t) \int_{0}^{t} T_{s} f d s$ does not converge a.e. as $t \rightarrow 0_{+}$.

Proof. Let $p$ fixed, $1<p<+\infty$. Consider the one-parameter semigroup $T_{t}$ defined by

$$
T_{t}\left(\sum_{n=1}^{\infty} \alpha_{n} \tilde{\phi}_{\pi(n)}\right)=\sum_{n=1}^{\infty} e^{i \lambda_{n} t} \alpha_{n} \tilde{\phi}_{\pi(n)}
$$

where the $\lambda_{n}$ are those of the previous lemma and $\pi$ the permutation in Theorem 1. Then we have $T_{0}=I$ (identity),

(i) for each $t, s, T_{t} \circ T_{s}=T_{t+s}$,

(ii) $\lim _{s \rightarrow t}\left\|T_{s} f-T_{t} f\right\|_{p}=0 \forall t \geqslant 0$ and $\forall f \in L P$,

(iii) $S_{t} f=\sum_{n=1}^{\infty}\left(1 / i \lambda_{n}\right)\left(\exp \left(-i \lambda_{n} t\right)-1\right) \alpha_{n} \phi_{\pi(n)}(t>0)$.

It is now easy to see that $\left(S_{K} / t_{K}\right) f$ does not converge almost everywhere for the function $f$ of Theorem 1 .

REMARK 7. It is known that for $T_{0}=I$ and any semigroup of positive bounded linear operators on $L^{p}$ with $P(\Omega)<+\infty$ we can get the local ergodic theorem (see [8]).

\section{REFERENCES}

1. A. Ionescu Tulcea, Ergodic properties of isometries in $L_{p}$ spaces, Bull. Amer. Math. Soc. 70 (1964), 366-371.

2. R. V. Chacon and S. A. McGrath, Estimates of positive contractions, Pacific J. Math. 30 (1969), 609-620.

3. M. A. Akcoglu, A pointwise ergodic theorem in $L^{p}$-spaces, Canad J. Math. 27 (1975), 1075-1082.

4. A. de la Torre, Simple proof of the maximal ergodic theorem, Canad. J. Math. 28 (1976), 1073-1075.

5. D. L. Burkholder, Semi-gaussian subspaces, Trans. Amer. Math. Soc. 104 (1962), 123-131.

6. M. A. Akcoglu and L. Sucheston, Remarks on dilations in $L_{p}$ spaces, Proc. Amer. Math. Soc. 53 (1975), 80-82.

7. M. Feder, On power-bounded operators and the pointwise ergodic property, Proc. Amer. Math. Soc. 83 (1981), 349-353.

8. U. Krengel, Monograph in preparation.

9. M. A. Akcoglu and U. Krengel, Two examples of local ergodic divergence, Israel J. Math. 33 (1979), 225-230.

10. P. L. Ulyanov, Divergent Fourier series, Uspekhi Mat. Nauk 16 (1961), 61-142, MR 23 A2701 = Russian Math. Surveys 16 (1961), 3-75.

11. Kolmogorov and divergent Fourier series, Uspekhi Mat. Nauk 38 (1983), 51-90 = Russian Math. Surveys 38 (1983), 57-100. 
12. M. A. Akcoglu, Pointwise ergodic theorems in $L_{p}$ spaces, Ergodic Theory Math. Forschungsinst. (Proc. Conf. Ergodic Theory Oberwolfach, 1978), Lecture Notes in Math., vol. 729, Springer-Verlag, Berlin, 1979, pp. 13-15.

13. I. Assani, Sur la convergence ponctuelle de quelques suites d'opérateurs (a paraître).

14. Sur les opérateurs à puissances bornées et le théorème ergodique ponctuel dans $L^{p}[0,1]$, $1<p<+\infty$ (a paraître).

15. A. M. Olevskii, Fourier series with respect to general orthogonal systems, Springer-Verlag, Berlin and New York, 1975.

Universite Paris Vi, laboratoire de Probabilites, Tour 56, 4 Place Jussieu, 75230 Paris Cedex 05, France 